\title{
FEDERATED CRITICAL INFRASTRUCTURE SIMULATORS: TOWARDS ONTOLOGIES FOR SUPPORT OF COLLABORATION
}

\author{
Katarina Grolinger, Miriam A.M. Capretz, Adam Shypanski, Gagandeep S. Gill \\ Department of Electrical and Computer Engineering, Faculty of Engineering \\ The University of Western Ontario \\ London, ON, Canada N6A 5B9 \\ \{kgroling, mcapretz, ashypans, ggill53\}@uwo.ca
}

\begin{abstract}
Our society relies greatly on a variety of critical infrastructures $(\mathrm{CI})$, such as power system networks, water distribution, oil and natural gas systems, telecommunication networks and others. Interdependency between those systems is high and may result in cascading failures spanning different infrastructures. Behavior of each CI can be observed and analyzed through the use of domain simulators, but this does not account for their interdependency. To explore CI interdependencies, domain simulators need to be integrated in a federation where they can collaborate.

This paper explores three different simulators: the EPANET water distribution simulator, the PSCAD power system simulator and the I2Sim infrastructure interdependency simulator. Each simulator's modeling approach is explored and their similarities and differences between modeling approaches are determined. Core ontology for each simulation engine is created as well as initial mapping between them. Ontologies and their mapping will support collaboration of simulators by enabling exchange of information in a semantic manner.
\end{abstract}

Index Terms - Ontology, Infrastructure Simulators, Critical Infrastructure interdependencies, Disaster Management.

\section{INTRODUCTION}

In the United States of America, critical infrastructure (CI) is defined as independent, man-made systems and processes that function collaboratively and synergistically to produce and distribute a continuous flow of essential goods and services [1]. The definition includes eight infrastructures: telecommunications, electric power systems, natural gas and oil, banking and finance, transportation, water supply system, government services and emergency services. In the European Union, CI is defined as an asset, system or part, which is essential for the maintenance of vital social wellbeing of people and includes three main sectors: energy, transport and information and communication technology [2]. The definitions emphasize the significance of CI for our society and our continuous, daily reliance on them. The US definition recognizes that the infrastructures are not isolated entities, performing independently, but are highly interdependent systems where successful delivery of goods and services is only possible through their collaboration. Due to high interdependencies, failure in one of the systems may result in cascading failures spanning different infrastructures.

Behavior of each $\mathrm{CI}$ is commonly observed and analyzed through the use of domain simulators. Each one of these domain simulators is a powerful tool for exploring behavior of each CI autonomously, but they do not account for interdependencies between infrastructures. To explore CI behavior in a more realistic scenario, interdependencies need to be included.

This paper explores three different simulators: the EPANET [3] water distribution simulator, the PSCAD [4] power system simulator and the I2Sim [5] infrastructure interdependency simulator. Core modeling ontology, as well as the initial ontology mapping between each simulator is created. Ontologies and their mapping will support the collaboration of simulators by enabling the exchange of information between simulators in a semantic manner.

This paper is organized as follows: Section 2 reviews related work regarding integration of simulators and ontologies' role in integration. Section 3 analyzes modeling approaches for the three simulators and defines the modeling ontology of each simulator. The ontology mapping approach is described in Section 4, while conclusions and future work are presented in Section 5.

\section{RELATED WORK}

Methods for the simulation of infrastructure interdependencies can be categorized into two groups. The first group, commonly referred to as federated simulators, attempts to integrate existing domain simulators by enabling their coordination and collaboration. DIESIS and EPOCHS belong to that category. The second group includes 
simulation frameworks that enable the modeling of different infrastructures and their interdependencies. CIMS and I2Sim belong to this category.

The DIESIS project (Design of an Interoperable European federated Simulation network for critical InfraStructures) aims to establish a basis for the modeling and simulation of CI based upon open standards. Usov et al. [6] describe integration architecture including relations between simulation engines, DIESIS communication layer and ontology components. The DIESIS methodology uses the ontological framework to isolate the description layer from the technological simulator layer.

Hopkinson et al. [7] propose the use of the electric power and communication synchronizing simulator (EPOCHS). EPOCHS integrates the PSCAD/EMTDC electromagnetic transient simulator, the PSLF electromechanical transient simulator, and the Network Simulator 2 (NS2). Simulation engines are integrated using only their built-in application programming interfaces (APIs).

Dudenhoeffer et al. propose the CIMS framework for infrastructure interdependency modeling and analysis [8]. The CIMS framework is based on the agent model where an agent is an individual entity that possesses behavior rules, senses its environment, and acts upon the environment by modifying its internal state and/or the environment. Within the CIMS framework each network is modeled as a connected graph of nodes and edges which represent connections between the nodes. Nodes also represent interdependencies between CI.

Casalicchio et al. propose the CRESCO framework [9] that attempts to combine the two approaches: federated domain simulators and interdependency simulators. CRESCO attempts to integrate domain simulators by using an integration simulation framework. Two different complementary implementations are presented: FederatedABMS and CISIA. In FederatedABMS each agent models an infrastructure while interdependencies are represented by exchanges between agents. CISIA breaks the model into components that are characterized by their ability to perform a task. A single component commonly represent elements from different domain simulators.

The Infrastructure Interdependency Simulator (I2Sim) uses a cell-channel model to represent the physical elements of $\mathrm{CI}$ and their interdependencies [5]. It enables the modeling of interdependencies without modeling the details of involved entities.

\section{SIMULATORS AND THEIR ONTOLOGIES}

In this work we explore three simulators, I2Sim, PSCAD and EPANET, focusing specifically on the modeling entities. Understanding how each simulator models the world is the starting point in creating ontologies for the integration of simulators.

\subsection{I2Sim modeling ontology}

I2Sim uses a cell-channel model where cells, channels and tokens are the main modeling entities. Cells perform a function transforming inputs to outputs. Tokens are units transported from one cell to another, and include things such as electricity, gas and people. Channels are links between cells through which tokens may flow, such as pipes, wires and streets.

Figure 1 depicts the I2Sim modeling ontology. Any modeling element is a component. Components are further classified into categories: Cells, Controls and Channels. Even though tokens are significant elements in I2Sim, they are not included in this modeling ontology since they are not modeling entities, but are used to explain and quantify the flow through the system. Controls are often considered a special cell, but we have chosen to represent them as a separate category due to the specific function they perform. While cells transform inputs to outputs by applying some function, controls only change the direction of a token flow by either splitting the flow in two directions or more (distributors) or by merging the flow of several channels into one (aggregators). Cells are further classified into: source cells, production cells, storage and modifier cells. Each cell group performs a specific function. Channels include simple channels and delay channels.

The two entities that are not directly related to entity in the real world are the I2Sim Probes and the Visualization panels. I2Sim Probes are elements placed in the simulation model to measure the flow of tokens in channels or to measure the inputs and outputs of cells and controls. Visualization panels are entities used to visualize the output of I2Sim probes.

The bottom of the diagram in Figure 1 displays the common concepts that will serve as the upper level ontology for relating the three simulator models. Concepts include cells, controls, channels, meters and others. Cells, controls and channels, are entities directly related to real word, while the last two, meters and others, are entities that may exist only in the simulation model, but are significant in analyzing infrastructure behavior. To illustrate the relationship between the modeling ontologies of I2Sim, EPANET and PSCAD, the bottom part of the ontology diagram will contain the same components for the three simulators' ontologies.

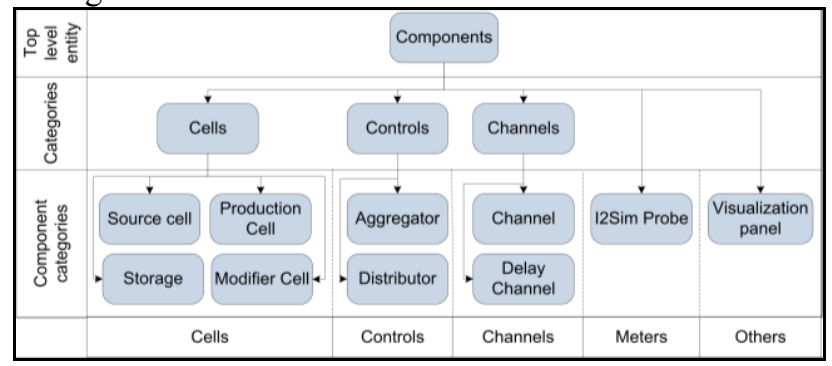

Fig. 1. I2Sim modeling ontology 


\subsection{EPANET modeling ontology}

Figure 2 illustrates the EPANET modeling ontology. In the category level there is significant similarity to the I2Sim modeling ontology. In EPANET nodes are entities that perform the transformation of inputs to outputs and include components such as reservoirs, tanks, junctions and controls. Nodes include two entities from the I2Sim model: cells and controls. EPANET links, since EPANET is water distribution simulator, transport water from one node to another and are simply a different term for channels from I2Sim.

Contrary to I2Sim, EPANET does not have specific entities to monitor the flow of tokens at individual points, instead the water flow in all nodes is automatically captured. The components that belong to the others category, such as labels, patterns and curves, are entities that simplify the water network modeling and add to its visualization.

\subsection{PSCAD modeling ontology}

Figure 3 represents PSCAD modeling ontology. Unlike I2Sim and EPANET, PSCAD does not have a middle layer

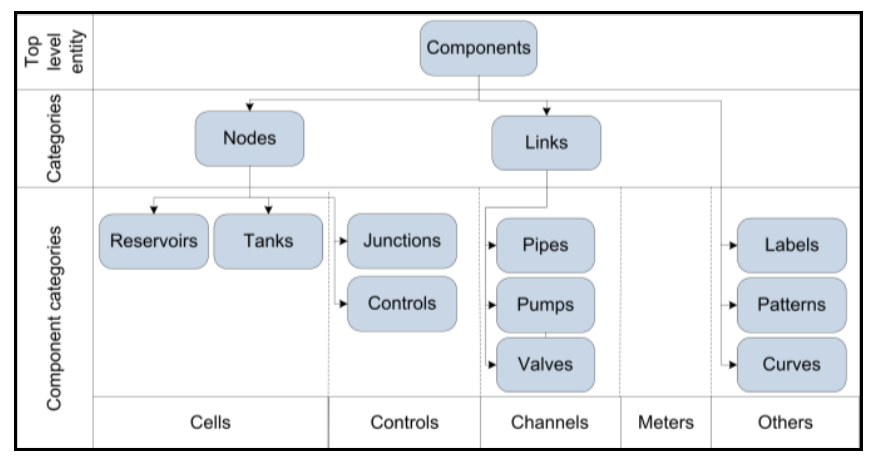

Fig. 2. EPANET modeling ontology to classify components into broader, generic categories. PSCAD starts from a variety of component categories specific for electrical distribution networks. Additionally, PSCAD has an additional layer for component subcategories, that neither I2Sim nor EPANET have, which represents a further specification for each component.

After analyzing the functionality of the PSCAD components, it is realized that they can clearly be grouped into the same component categories as I2Sim or EPANET: cells, channels, controls, meters and others (Fig. 3).

The cell category contains a wide variety of electric components that perform different functions. The channels category is made of components that transmit electricity: transmission lines and cables. Opposed to I2Sim which has only one measuring entity, the I2Sim Probe, and EPANET which does not have a specific measuring entity; PSCAD has very developed notion of meters. It offers a variety of meters for measurement of different properties of the electrical network distribution.

\section{ONTOLOGY MAPPING}

The three observed simulators have very different purposes and as such contain a variety of modeling entities that are domain specific. Therefore we cannot map all elements from one simulator to elements of another one. We have chosen to use an upper ontology that represents a generalized view of the three modeling ontologies. Concepts of each domain ontology are mapped to this upper ontology that acts as mediator. When illustrating the modeling ontologies of the three simulators in Section 3, the modeling entities were categorized into five categories: cells, controls, channels, meters and others to represent the relation between the three ontologies. This is shown at the bottom part of the diagrams in Figures 1,2 and 3. Those entities become concepts of the upper ontology.

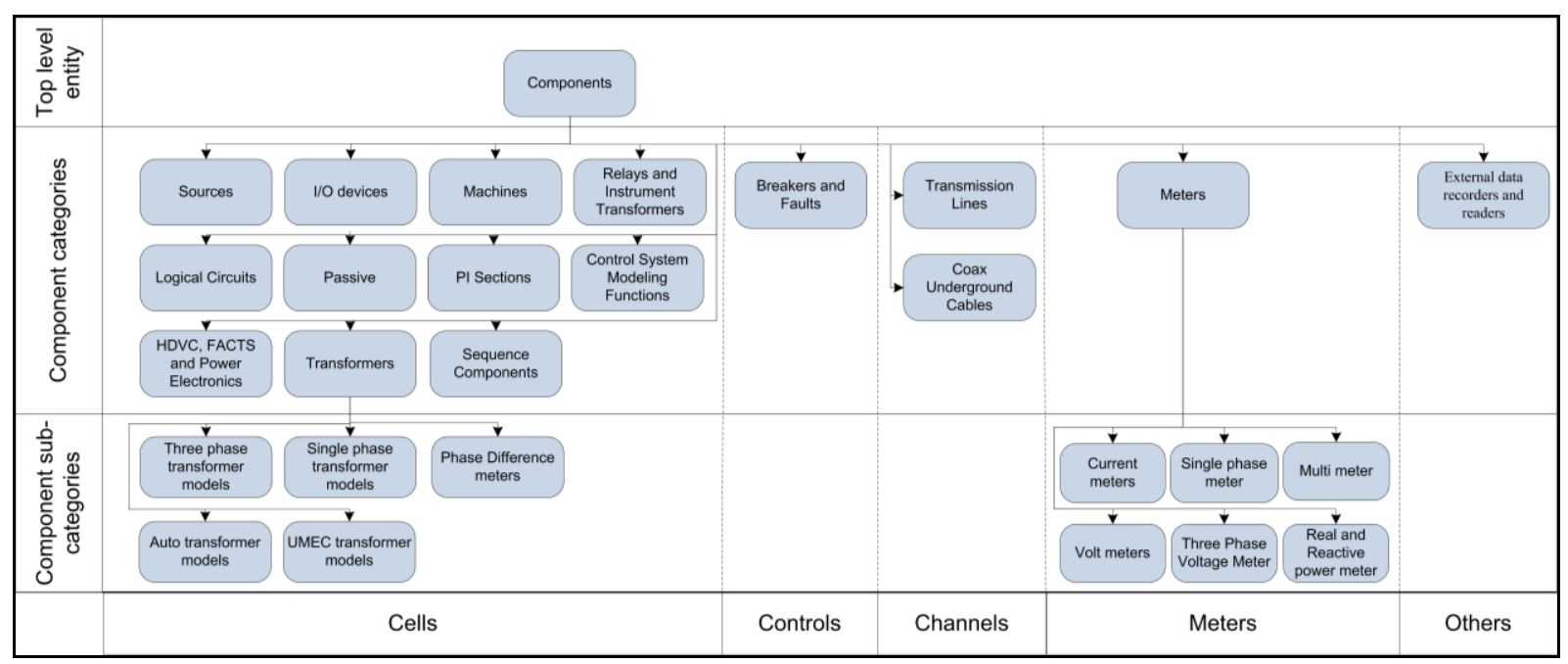

Fig. 3. PSCAD modeling ontology 
Figures 4, 5 and 6 illustrate the main concepts from the upper ontology and the mapping between the upper ontology and the I2Sim, EPANET and PSCAD modeling ontologies. Only the component category layer is mapped while entities from the middle layer of the modeling ontologies are not. Some of the lower layers in the I2Sim and PSCAD concepts, such as the I2Sim probes and the PSCAD labels, do not have a related middle layer concept. Moreover, PSCAD does not have a middle layer of categories at all.

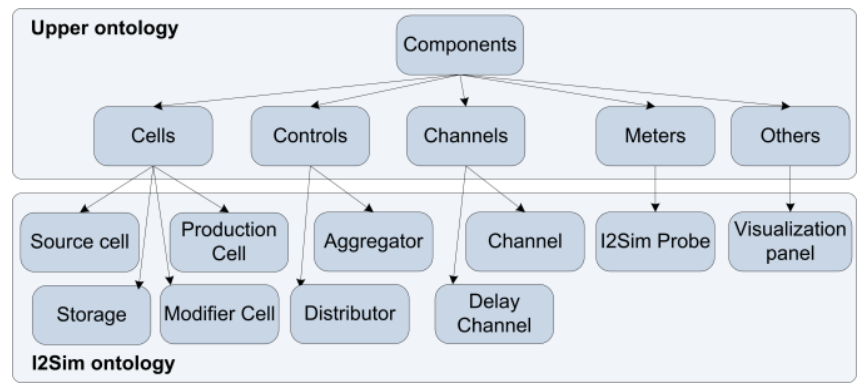

Fig. 4. I2Sim ontology mapping

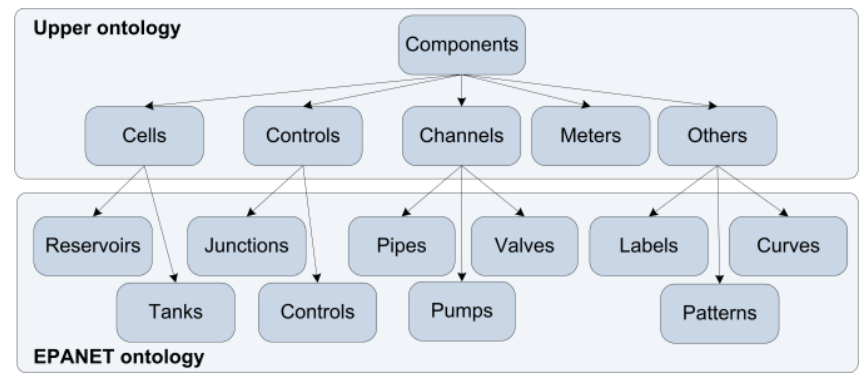

Fig.5. EPANET ontology mapping

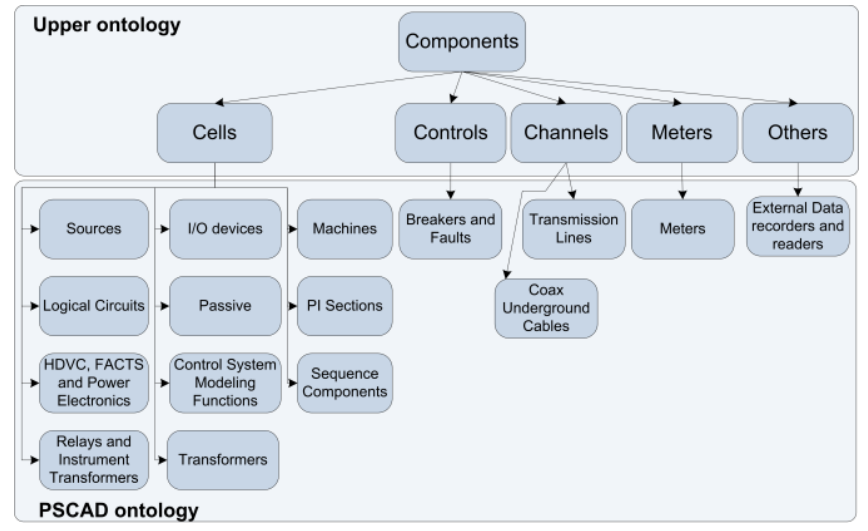

Fig. 6. PSCAD ontology mapping

\section{CONCLUSIONS AND FUTURE WORK}

The behavior of different critical infrastructures (CI) is commonly observed through the use of domain specific simulators. Even though they are very powerful tools in their respective domains, they do not account for CI interdependencies. Interdependency simulators, such as I2Sim, model interdependencies without modeling the details of each infrastructure. Combining the interdependency simulators with domain simulators would enable harnessing the power of both.

We explored modeling approaches of three different simulators: the EPANET water distribution simulator, the PSCAD power system simulator and the I2Sim infrastructure interdependency simulator. Even though the domains are quite different, our analysis showed similarities between the three modeling approaches. For the three simulators, the modeling entities can be categorized into five categories: cells, controls, channels, meters and others.

Mapping between the three modeling ontologies was done though the use of the upper ontology. Concepts from each simulator's ontology were mapped to the upper ontology that serves as the mediator between the simulators ontologies. The ontology and the mapping will support exchange of information between the simulators.

The next step will be to identify properties of each simulator's concepts that are relevant for the exchange of information between simulators, as well as their mapping.

\section{REFERENCES}

[1] Critical foundation: Protecting America's infrastructures, http://www.iwar.org.uk/cip/resources/pccip/report index.html. [2] European Commission, "Council Directive 2008/114/EC of 8 December 2008 on the Identification and Designation of European Critical Infrastructures and the Assessment of the Need to Improve Protection," 2008.

[3] EPANET, Water Distribution Modeling, http://www.epa.gov.proxy2.lib.uwo.ca:2048/nrmrl/wswrd/dw/epan et.html.

[4] PSCAD - power system simulator, https://pscad.com/products/pscad/.

[5] H.A. Rahman, M. Armstrong, D. Mao, J.R. Marti, "I2Sim: A Matrix-Partition Based Framework for Critical Infrastructure

Interdependencies Simulation," Proceedings of the Electric Power Conference, 2008, pp. 1-8.

[6] A. Usov, C. Beyel, E. Rome, U. Beyer, E. Castorini, P. Palazzari, et al., "The DIESIS Approach to Semantically Interoperable Federated Critical Infrastructure Simulation," Proceedings of the Second International Conference on Advances in System Simulation, 2010, pp. 121-128.

[7] K. Hopkinson, X. Wang, R. Giovanini, J. Thorp, K. Birman, D. Coury. "EPOCHS: A Platform for Agent-Based Electric Power and Communication Simulation Built from Commercial Off-the-Shelf

Components," IEEE Transactions on Power Systems, vol. 21, Issue 2, 2006, pp. 548-558.

[8] D.D. Dudenhoeffer, M.R. Permann, M. Manic, "CIMS: A Framework for Infrastructure Interdependency Modeling and Analysis," Proceedings of the Winter Simulation Conference, 2006, pp. 478-485.

[9] E. Casalicchio, R. Setola, S. Bologna, "A Two-Stage Approach to Simulate Interdependent Critical Infrastructures," Complexity in Engineering, 2010, pp. 76-78. 